\title{
Surface Plasmon Polariton Coupling between Nano Recording Marks and their Effect on Optical Read-out Signal
}

\author{
Kuo Pin Chiu ${ }^{1}$, Kuei Fu LAI ${ }^{1}$, Shih Chiang YeN $^{1}$, and Din Ping Tsai ${ }^{1,2,3 *}$ \\ ${ }^{1}$ Department of Physics, National Taiwan University, Taipei 10617, Taiwan, R.O.C. \\ ${ }^{2}$ Research Center for Applied Sciences, Academia Sinica, Taipei 11529, Taiwan, R.O.C. \\ ${ }^{3}$ Instrument Technology Reserch Center (ITRC), National Applied Research Laboratories, Hinchu 300, Taiwan, R.O.C.
}

(Received July 14, 2008; Accepted March 23, 2009)

\begin{abstract}
We use the three-dimensional finite-difference time-domain method to investigate surface plasmon polariton coupling between two nano-recording marks which are of different shapes. The different coupling characteristics and the influence of these coupling effects on the read-out reflection signal will be discussed. (C) 2009 The Optical Society of Japan
\end{abstract}

Keywords: optical data storage, surface plasmon polariton, near-field coupling, nano-recording mark, 3D FDTD

\section{Introduction}

In the fields of optical data storage, phase-change materials are used as the recording layer because of the reflection difference between amorphous and crystalline states of these materials. ${ }^{1,2)}$ Using a laser light source to heat up the phase-change material, it is possible to write down crystalline-state recording marks with different pit lengths in amorphous-state surroundings. ${ }^{3,4)}$ These length-modulated recording marks will connote digital information which can be read out by detecting the optical reflection signals. To approach the goal of increasing the information storage capacity of an optical disk, reducing the recording mark size is a straightforward and commonly used method. However, when the size of the recording mark becomes smaller and smaller, the difference of the reflection signal from marks and surroundings will become difficult to recognize. Many types of super-resolution near-field optical nanostructure disks have been proposed to improve the reflection signal contrast or carrier-to-noise-ratio (CNR) of an optical disk with sub-wavelength recording marks. ${ }^{5-8)}$ Although the improved CNR signals of sub-wavelength recording marks in these optical disks have been demonstrated experimentally, explanations of the super-resolution effect in those which have different near-field active layers are still not completely clarified (different mechanisms have been proposed for different types of active layer). ${ }^{6,7,9,10)}$ Among them, the near-field plasmonic coupling between recording marks in the phase-change layer and metallic nanostructures in the active layer is adopted as one of the key factors responsible for the optical super-resolution effects. The plasmonic coupling effect between metallic nanostructures can localize and enhance the light field around them, and their optical resonances are strongly dependent on structure geometry. These properties of plasmonic nanostructures provide great freedom for people to use them in many nanophotonic applications, such as tuning field enhancement or confinement, ${ }^{11-13)}$ controlling absorption or extinction, ${ }^{11,14)}$ and modulating resonance modes. ${ }^{13,15,16)}$

\footnotetext{
*E-mail address: dptsai@phys.ntu.edu.tw
}

On the other hand, since the high density region of a laser focusing spot at the focus distributes itself ever a spheroidlike region, it is possible to write down two crystalline-state recording marks simultaneously in two vertically separated phase-change material layers. The crystalline-state of a phase-change material possesses similar optical properties to a metal in which the real part of dielectric constant is negative, and they can be formed in different sizes and shapes by controlling the power of the incident laser beam. Therefore, these two marks can optically interact with each other when another laser focusing spot is used to read them out. In view of the near-field plasmonic coupling mechanism to realize the super-resolution effect and the metal-like material property of crystalline- $\mathrm{Ge}_{2} \mathrm{Sb}_{2} \mathrm{Te}_{5}\left(\mathrm{cr}_{-} \mathrm{Ge}_{2} \mathrm{Sb}_{2} \mathrm{Te}_{5}\right)$ alloy, we wanted to see what happened if we replaced the active layer with another phase-change layer in the optical disk structure, and we wanted to discuss the influence of near-field surface plasmon polariton (SPP) coupling between two recording marks on optical reflection signals. In this paper, we numerically calculated the near-field SPP coupling effect between two nano-recording marks with different shapes. The characteristics of these coupling effects and their influence on reflected read-out signal of an optical disk are discussed.

\section{Model of Computation}

\subsection{Computation method}

In the following computations, the three-dimensional finite difference time domain (3D FDTD) method is used to calculate the near-field optical responses of the system. The spatial grid size and full computation space are set to $5.0 \times 5.0 \times 5.0 \mathrm{~nm}^{3}$ and $1.6 \times 1.6 \times 0.5 \mu \mathrm{m}^{3}$, respectively, and a perfect match layer (PML) is used as the simulation boundary condition. A continuous light source with Gaussian profile and wavelength $(\lambda) 658 \mathrm{~nm}$ is used as an incident light, which is circularly polarized and propagates along the $z$-direction relative to the sample structure.

\subsection{Sample structure and material parameters}

Figure 1(a) shows a schematic diagram of the sample structure for our computations. Light is incident from the bottom of the sample and then passing through the layered 


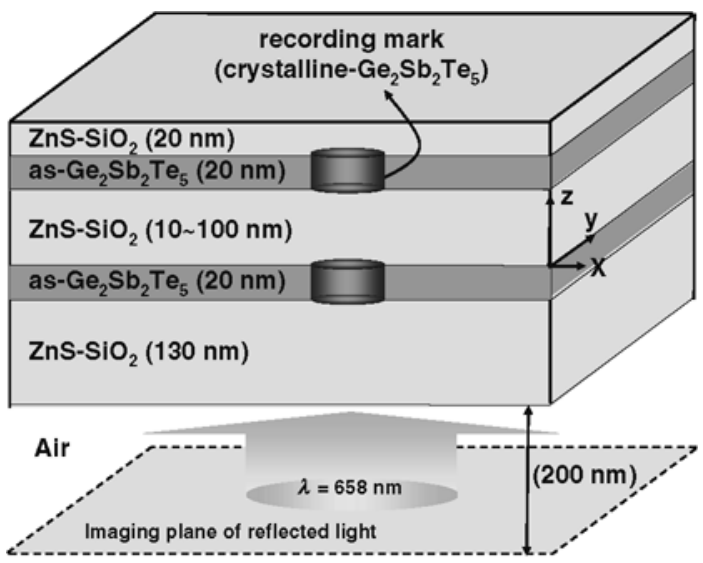

(a)

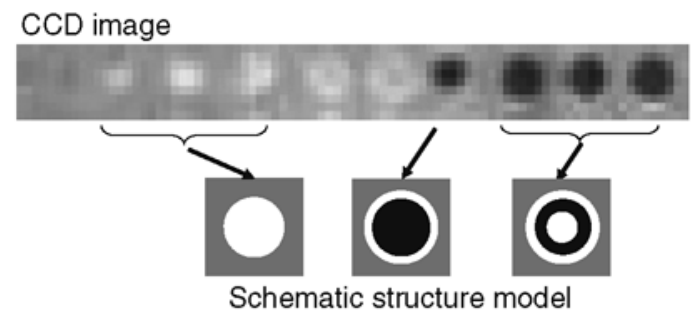

(b)

Fig. 1. (a) Schematic diagram of the sample structure for numerical investigation. (b) CCD images of reflection light from different types of recording mark which are produced by illuminating different laser writing powers on phase-change material.

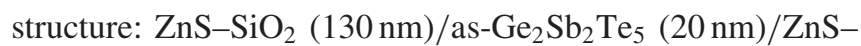

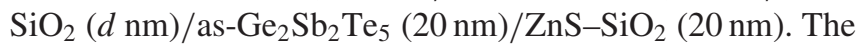
as- $\mathrm{Ge}_{2} \mathrm{Sb}_{2} \mathrm{Te}_{5}$ denotes asdeposited- $\mathrm{Ge}_{2} \mathrm{Sb}_{2} \mathrm{Te}_{5}$ which is an initial state of the phase-change material just after sputtering. The two recording marks depicted in Fig. 1(a) are cr$\mathrm{Ge}_{2} \mathrm{Sb}_{2} \mathrm{Te}_{5}$ which may form in different shapes when using different laser power to record the marks. ${ }^{17}$ The spacer layer, $\mathrm{ZnS}-\mathrm{SiO}_{2}$, between two as- $\mathrm{Ge}_{2} \mathrm{Sb}_{2} \mathrm{Te}_{5}$ layers is used to control the separation distance, $d$, of the two recording marks and its thickness is varied from $10 \mathrm{~nm}$ to $100 \mathrm{~nm}$ in our computations. Figure 1(b) shows some measured CCD images of the reflected light from the recording marks (upper pictures) which are produced by different laser writing powers. Because the crystalline-state $\mathrm{Ge}_{2} \mathrm{Sb}_{2} \mathrm{Te}_{5}$ (recording mark) has higher reflectivity than that of the asdepositedstate $\mathrm{Ge}_{2} \mathrm{Sb}_{2} \mathrm{Te}_{5}$ (the surroundings), we modeled three types of recording marks shown in the lower part of Fig. 1(b). The white and gray regions are $\mathrm{cr}-\mathrm{Ge}_{2} \mathrm{Sb}_{2} \mathrm{Te}_{5}$ and as- $\mathrm{Ge}_{2} \mathrm{Sb}_{2} \mathrm{Te}_{5}$, respectively, and the dark region is just air.

\subsection{Material parameters}

The optical constants (refraction index, $n$, and extinction coefficient, $\kappa$ ) of each material used in our computations are listed in Table 1. These material parameters are measured experimentally by an ellipsometer except that the optical constants of $\mathrm{cr}-\mathrm{Ge}_{2} \mathrm{Sb}_{2} \mathrm{Te}_{5}$ are referred to the paper of Kim et al. ${ }^{18)}$
Table 1. Optical constants of thin films of materials (for $\lambda=658 \mathrm{~nm})$.

\begin{tabular}{lcc}
\hline \multicolumn{1}{c}{ Material } & $n$ & $\kappa$ \\
\hline $\mathrm{as}_{-}-\mathrm{Ge}_{2} \mathrm{Sb}_{2} \mathrm{Te}_{5}$ & 4.13 & 2.07 \\
$\mathrm{cr}-\mathrm{Ge}_{2} \mathrm{Sb}_{2} \mathrm{Te}_{5}$ & 3.06 & 4.28 \\
$\mathrm{ZnS}-\mathrm{SiO}_{2}$ & 2.15 & 0.00 \\
\hline
\end{tabular}

\section{Results and Discussion}

\subsection{Reflection signals of disk-shaped marks}

Figure 2 shows the variations of maximum intensity [Fig. 2(a)] and full-width at half-maximum (FWHM) [Fig. 2(b)] of the reflected light in the imaging plane [as depicted in Fig. 1(a)] with different spacer thickness, $d$, for disk-shaped recording marks. The cases denoted by twomarks mean that there is a recording mark in both as$\mathrm{Ge}_{2} \mathrm{Sb}_{2} \mathrm{Te}_{5}$ layers of the sample structure, and that denoted by one-mark mean that there is one recording mark in the first as- $\mathrm{Ge}_{2} \mathrm{Sb}_{2} \mathrm{Te}_{5}$ layer (close to the light source) only. The diameter, $D$, of each mark is changed from 100 to $400 \mathrm{~nm}$ in our computations. From the results shown in Fig. 2(a), we see first that the reflected light intensity gradually increases when the diameter of recording mark increases for both the two- and the one-mark cases. This is due to the raised portion of high reflection $\mathrm{cr}-\mathrm{Ge}_{2} \mathrm{Sb}_{2} \mathrm{Te}_{5}$ in the phase-change layers. We also can see that there is a small reduction in reflected intensity for the two-mark case compared to that of the one-mark case, which probably resulted from the coupling effect between the two recording marks that trapped more light energy in the near-field. This coupling effect leads to a decrease in intensity and increase in FWHM of the reflected light. From the results of Figs. 2(a) and 2(b), it is seen that the coupling effect between the two recording marks might be stronger when the spacer thickness is about 40 and $90 \mathrm{~nm}$ at which the changes in reflection intensity or FWHM are more distinct. For a clearer representation, we show in Fig. 2(c) the reflected intensity difference between the one-mark and the two-mark cases of Fig. 2(a). There are obviously two intensity difference peaks appearing at the spacer thickness described above. Besides these, Figs. 2(a) and 2(b) also indicate that the characters of coupling effect between the two recording marks may be different at spacer thicknesses around $40 \mathrm{~nm}$ and $90 \mathrm{~nm}$ because of the different variation behavior in reflected light. That is, the decrease in reflected intensity is more obvious (increase in FWHM is small) at the spacer thickness around the $40 \mathrm{~nm}$ region, and the increase in FWHM is more obvious (decrease in intensity is small) around the $90 \mathrm{~nm}$ region. Finally, the small discrepancy of reflection signals between the one- and two-mark cases also implies that the optical coupling or cross-interaction of the two recording marks may be weak for disk-shaped recording marks.

In order to see what happens in the near-field, we show the distributions of field intensity ratio (normalized by the corresponding values without any marks in the sample structure) around the two recording marks at spacer thicknesses of 40 and $90 \mathrm{~nm}$ in Fig. 3. The gray curves in each 


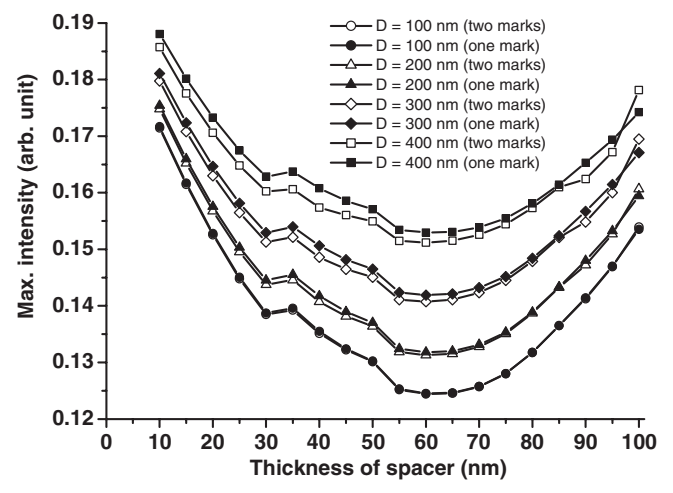

(a)

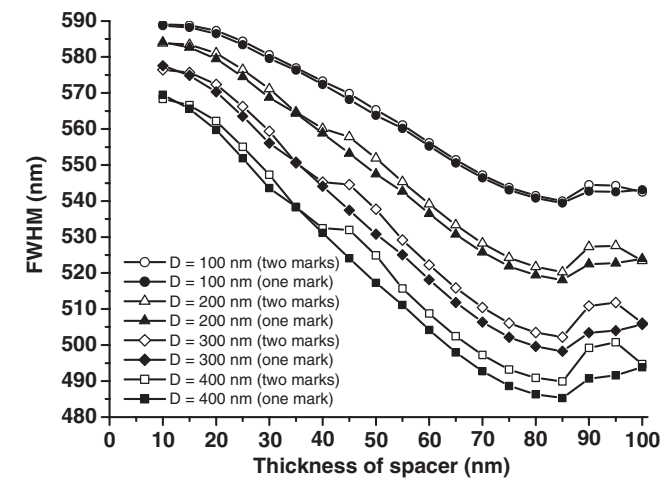

(b)

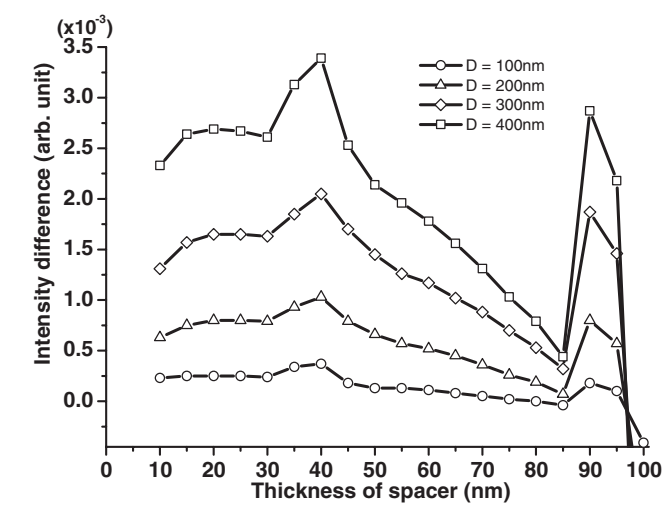

(c)

Fig. 2. Spacer thickness dependent variation of (a) maximum intensity and (b) FWHM of reflected light in the imaging plane for sample structures of one- and two-disk-mark cases. (c) The reflection intensity difference between one- and twodisk-mark cases.

segment of Fig. 3 represent the contours of the intensity ratio values. The white dashed lines and white rectangular lines in each figure indicate the coordinates of layered disk structure and location of the recording marks, respectively. Figures 3(a) and 3(b) are results for spacer thickness $40 \mathrm{~nm}$, and 3(c) and 3(d) are those for spacer thickness $90 \mathrm{~nm}$. These figures show that the field intensities are concentrated at the edges of the recording marks. They result from the field localization and enhancement effects of the SPP oscillations of the recording marks. ${ }^{19)}$ Comparing the results of the twoand one-mark cases, it is seen that more of the field intensity is trapped in between and around the mark edges in the twomark case, which results from the SPP coupling effect of the two marks. The relatively lower reflection signal for the twomark sample might be explained by this light trapping effect of SPP coupling between the two recording marks. On the other hand, it can be seen from the distributions of intensity ratio in Figs. 3(a) and 3(c) that the light coupling characters are different for spacer thickness at $40 \mathrm{~nm}$ and $90 \mathrm{~nm}$.

\subsection{Reflection signals of ring-shaped marks}

We also calculated the cases of sample structure with ring-shaped recording marks. The outer diameter, $D$, of the ring-mark is changed from 100 to $500 \mathrm{~nm}$, and the inner diameter of the mark is set to $0.6 D$. Figure 4 shows the variations of maximum intensity of the reflected light in the imaging plane with different spacer thickness. It is obvious that the variation tendencies of maximum reflection intensity with spacer thickness for two ring-marks and one ring-mark cases roughly behave contrary to each other when $D$ is larger than $300 \mathrm{~nm}$. That is, for the two ring-mark cases, the reflection intensity is roughly larger at large spacer thickness than at small spacer thickness, and it gradually increases as this thickness is increased when the spacer thickness larger than $40 \mathrm{~nm}$. However, for one ring-mark cases, the reflection intensity is roughly larger at small spacer thickness than at large spacer thickness, and it gradually decreases as this thickness is increased up to about $80 \mathrm{~nm}$. On the other hand, the variation tendencies of maximum reflection intensity with spacer thickness for both two and one ring-mark cases are similar to those of disk-shaped cases when $D$ is smaller than $200 \mathrm{~nm}$. In further analysis of these results, we infer that the different reflection behavior at large $D$ is a consequence of the Fabry-Perot effect of the sample structure. The reason for this inference will be described in the following.

Figure 5 shows the spacer thickness dependent reflectivity for four different layered structures which are calculated by transfer matrix method (TMM). The layered structure and material parameters used in this TMM calculation are the same as those used in FDTD simulations except that there is no recording mark and, also, the two as- $\mathrm{Ge}_{2} \mathrm{Sb}_{2} \mathrm{Te}_{5}$ layers are changed to different materials. Materials denoted before 


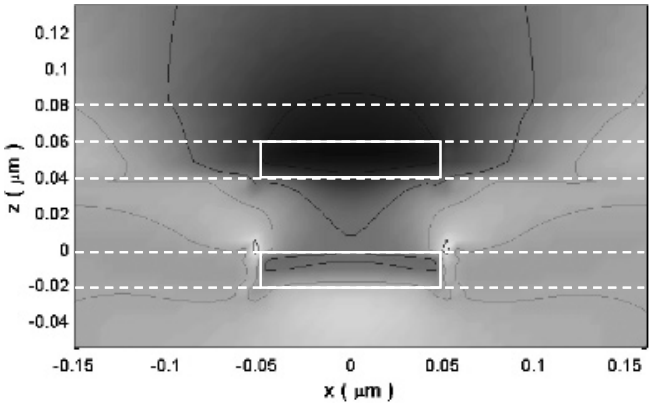

(a)

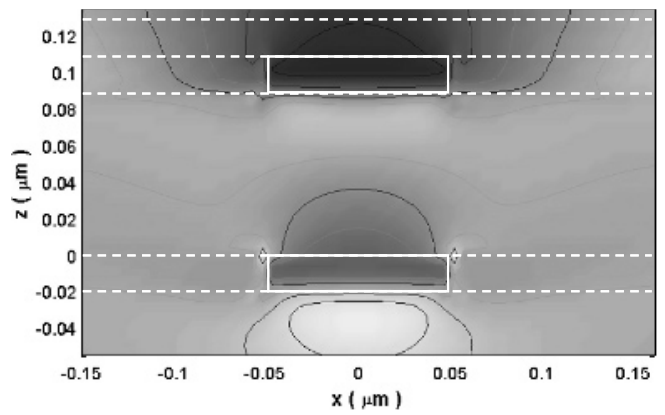

(c)

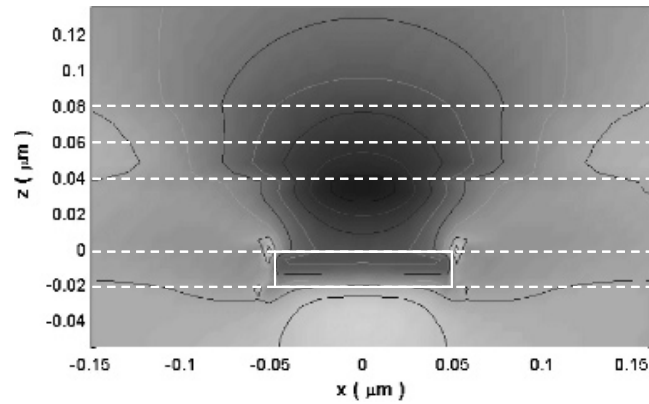

(b)

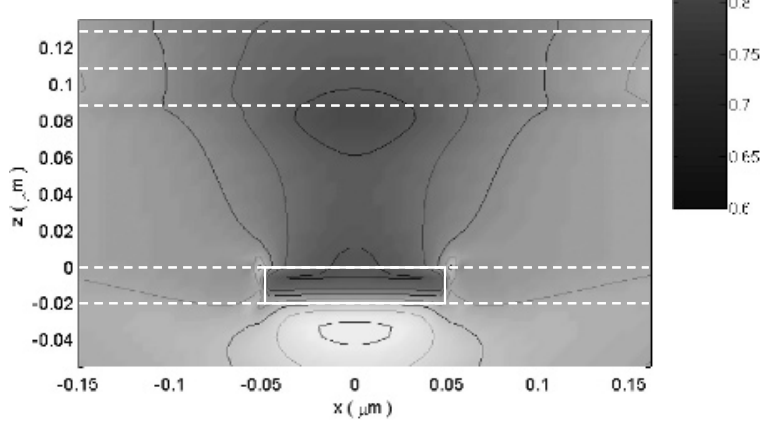

(d)

Fig. 3. Near-field intensity ratio distributions in $x-z$ cross-section of the sample structure when mark size $D=100 \mathrm{~nm}$. (a) two disk-mark case at spacer thickness $40 \mathrm{~nm}$, (b) one disk-mark case at spacer thickness $40 \mathrm{~nm}$, (c) two disk-mark case at spacer thickness $90 \mathrm{~nm}$, and (d) one disk-mark case at spacer thickness $90 \mathrm{~nm}$.

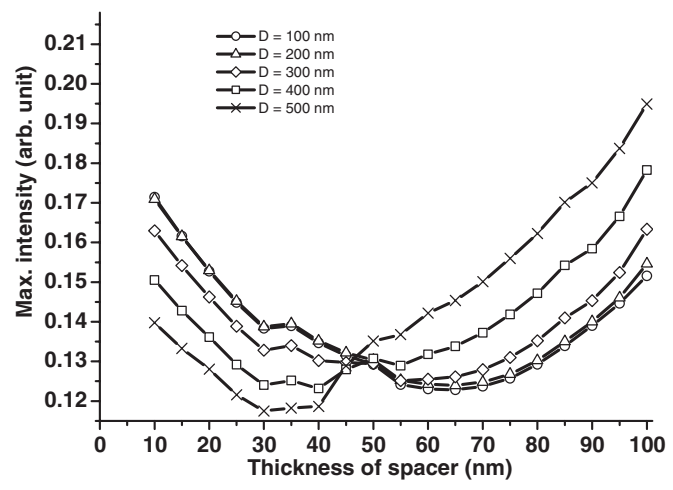

(a)

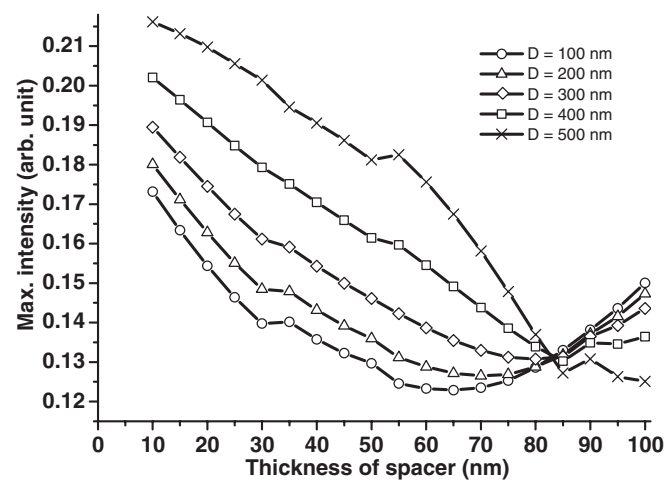

(b)

Fig. 4. Variations of maximum reflection intensity with spacer thickness for sample structures with (a) two ring-mark cases and (b) one ring-mark cases in the imaging plane.

and after the double slash line of the legend in Fig. 5 represent the materials used as the first and second as$\mathrm{Ge}_{2} \mathrm{Sb}_{2} \mathrm{Te}_{5}$ layers in the sample structure, respectively. From the results shown in that figure, we can see that the trend of the curve of large $D$ cases for two ring-marks in Fig. 4(a) is probably following the combined curve trends of as-GST// as-GST and Air//Air cases. And when the central air region increases with increase in the diameter of the ring-mark, the reflection character coming from the Air//Air layered structure becomes greater and greater. Therefore, the reflection intensity is gradually increasing for large spacer thickness. Similarly, the variation tendency of reflection intensity with spacer thickness of the one ring-mark case can be elucidated by the same argument, that is, by combining the curve trends of as-GST//as-GST and Air//as-GST cases in Fig. 5. Because the variations of reflectivity with spacer thickness for different layered structures appearing in Fig. 5 all result from the Fabry-Perot effect of the layered structure, we infer that the dominating curve trends of reflection intensity shown in Fig. 4 may result from the mixed Fabry-Perot effects of different materials in the sample structure. 


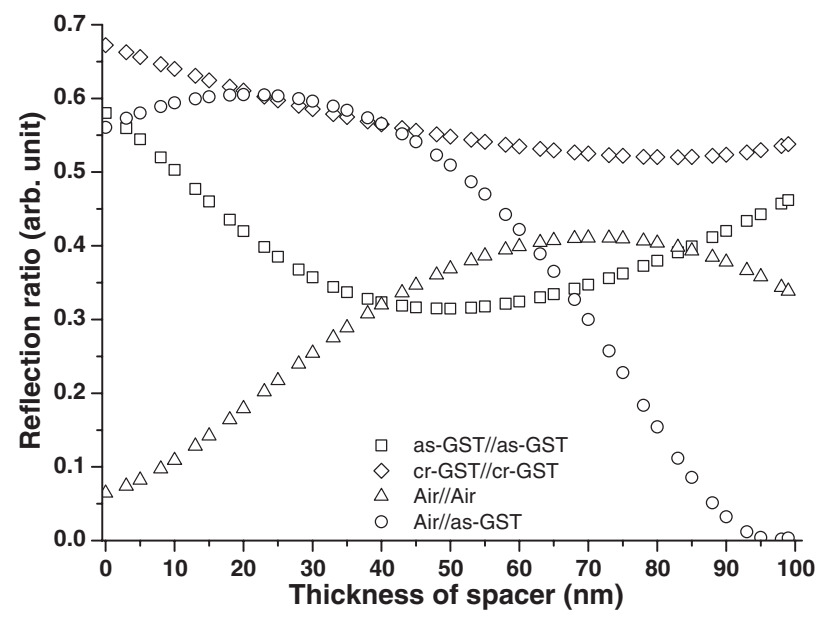

Fig. 5. Spacer thickness dependent reflectivity for four different layered structures calculated by transfer matrix method.

\subsection{Comparison of different shaped marks}

According to the results in Figs. 2 and 4, it is seen that for small mark size, the variation tendency of reflection intensity for each case is similar (it is dominated by the Fabry-Perot effect of as-GST//as-GST layered structure). However, there are still some differences between them, which are likely caused by different characters of SPP coupling between the recording marks. To diminish the influence of Fabry-Perot effect coming from different layered structures, we take the results of each mark type whose diameter is $100 \mathrm{~nm}$ for comparison. Here, we also put in the result of the third mark type (ring-sphere-shaped marks) as depicted in Fig. 1(b). For this type of recording mark, the sphere located at the center of the ring is $30 \mathrm{~nm}$ in diameter. Figures 6(a) and 6(b) show the variations of maximum field intensity ratio and total intensity ratio in the imaging plane for all three different types of recording marks with $D=100 \mathrm{~nm}$. The ratio here means that the values of reflected field intensities are divided by the corresponding values when there is no recording mark in each as- $\mathrm{Ge}_{2} \mathrm{Sb}_{2} \mathrm{Te}_{5}$ layer of the sample structure; and the total intensity here is the summation of intensity values over a circular area with a diameter of $560 \mathrm{~nm}$ in the imaging plane. Because the reflected light within this area can be collected by an objective with numerical aperture of 0.65 in the far-field, the total intensity ratio can be analogous to the CNR signal of the recording mark. We use these ratio values to extract the influence of the coupling effect between the recording marks.

It can be seen in both Figs. 6(a) and 6(b) that the ringshaped and ring-sphere-shaped recording marks has similar variation in reflection signals when the separation distance of the two marks is changed. This result implies that their near-field SPP coupling might be analogous to each other, at least in the small mark case. On the other hand, comparison of the results for ring-shaped and disk-shaped recording marks showed that the former exhibited smaller intensity in reflected light. This is because the SPP oscillation response of ring-shaped mark leads to higher field localization or

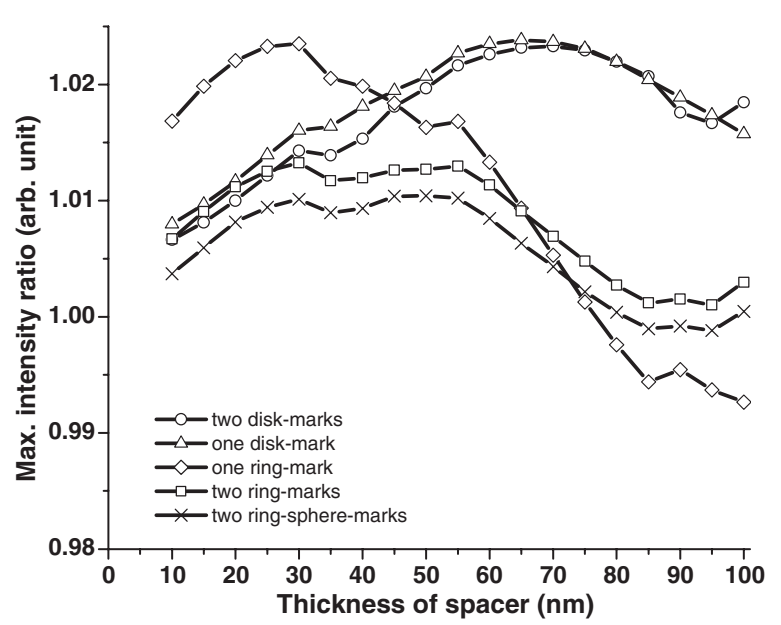

(a)

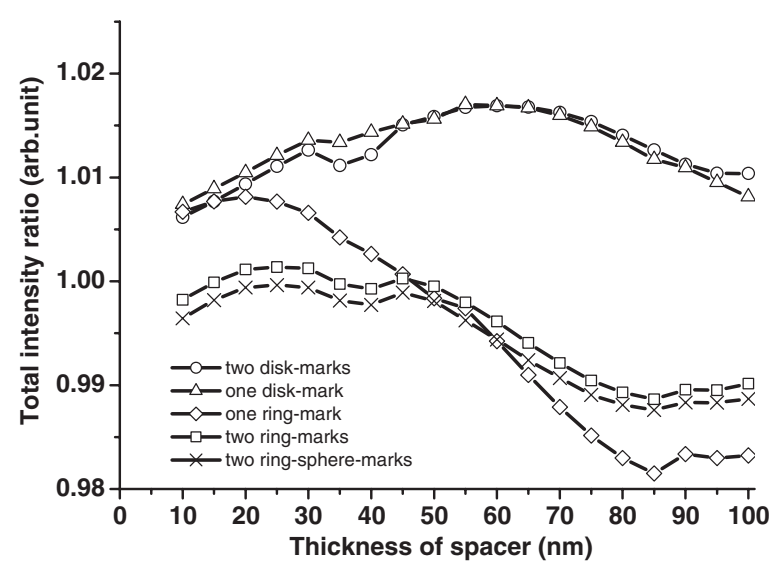

(b)

Fig. 6. Variations of (a) maximum field intensity ratio and (b) total intensity ratio in the imaging plane for all three different types of recording marks with $D=100 \mathrm{~nm}$.

trapping at the inner edges of the marks; the reflected light intensity is therefore weaker than that of the disk-shaped cases. Also, because the SPP coupling between the two recording marks in two-mark cases can trap more light energy around them comparing to that in one-mark cases, the former have lower reflection intensities than the latter when spacer thickness is small. Finally, we can see that the value difference between maximum and total intensity ratio is larger in ring-mark cases than in disk-mark cases, especially when the spacer thickness is less than $50 \mathrm{~nm}$. This means that the SPP coupling effects of ring-shaped recording marks will result in a more spatially concentrated field distribution than that of disk-shaped marks.

\section{Conclusions}

We have investigated the SPP coupling between two recording marks and their influence on the optical reflection signal in this paper. From the behaviors of reflection signals for different types of recording marks in the sample structure, the characteristics of the SPP coupling phenomenon which depend on either separation distance or geo- 
metrical shape of the recording marks have been discussed. We demonstrated that the SPP oscillations of two marks can trap light energy around the marks, and that the reflected field distribution is more spatially concentrated for ringshaped marks than that of disk-shaped marks when SPP coupling effect is strong. Our results also show that it may lead to a lower reflection signal ratio when the SPP coupling between the two marks is strong, which seems in conflict with that of super-resolution near-field optical structure disks. This may be due to the large extinction coefficients of the $\mathrm{cr}-\mathrm{Ge}_{2} \mathrm{Sb}_{2} \mathrm{Te}_{5}$ alloy so that light energy is largely consumed in these nanostructures when trapped by their SPP coupling.

\section{Acknowledgments}

The authors are grateful for the research support from the National Science Council of Taiwan, R.O.C., under project number NSC-97-2120-M-002-013-, NSC-96-2923-M-002-002-MY3, and the Ministry of Economic Affairs, Taiwan, R.O.C., under grant number 96-EC-17-A-09-S1-019, respectively. They are also grateful to the National Center for High-performance Computing for computer time and facilities, and also thank the Computer and Information Networking Center, National Taiwan University for supporting the high-performance computing facilities. D. P. Tsai is grateful for the support of the National Center for Theoretical Sciences, Taipei Office.

\section{References}

1) N. Yamada, E.Ohno, K. Nishiuchi, N. Akahira, and M. Takao: J. Appl. Phys. 69 (1991) 2849.

2) J. H. Coombs, A. P. J. M. Jongenelis, W. van Es-Spiekman, and B. A. J. Jacobs: J. Appl. Phys. 78 (1995) 4906.
3) M. Miyamoto, A. Hirotsune, Y. Miyauchi, K. Ando, M. Terao, N. Tokusyuku, and R. Tamura: IEEE J. Sel. Top. Quantum Electron. 4 (1998) 826.

4) T. Ohta: J. Optoelectron. Adv. Mater. 3 (2001) 609.

5) J. Tominaga, H. Fuji, A. Sato, T. Nakano, T. Fukaya, and N. Atoda: Jpn. J. Appl. Phys. 37 (1998) L1323.

6) L. Men, J. Tominaga, H. Fuji, and N. Atoda: Jpn. J. Appl. Phys. 39 (2000) 2639.

7) W. C. Lin, T. S. Kao, H. H. Chang, Y. H. Lin, Y. H. Fu, C. T. Wu, K. H. Chen, and D. P. Tsai: Jpn. J. Appl. Phys. 42 (2003) 1029.

8) H. Fuji, T. Kikukawa, and J. Tominaga: Jpn. J. Appl. Phys. 42 (2003) L589.

9) W. C. Liu and D. P. Tsai: Jpn. J. Appl. Phys. 42 (2003) 1031.

10) K. Katajai, J. Olkkonen, J. Aikio, and D. Howe: Jpn. J. Appl. Phys. 43 (2004) 160.

11) F. Hao, P. Nordlander, M. T. Burnett, and S. A. Maier: Phys. Rev. B 76 (2007) 245417.

12) S. I. Bozhevolnyi and T. Søndergaard: Opt. Express 15 (2007) 10869.

13) G. Pellegrini, V. Bello, G. Mattei, and P. Mazzoldi: Opt. Express 15 (2007) 10097.

14) A. P. Hibbins, W. A. Murray, J. Tyler, S. Wedge, W. L. Barnes, and J. R. Sambles: Phys. Rev. B 74 (2006) 073408.

15) G. Lévêque and Olivier J. F. Martin: Opt. Express 14 (2006) 9971.

16) A. Christ, T. Zentgraf, S. G. Tikhodeev, N. A. Gippius, J. Kuhl, and H. Giessen: Phys. Rev. B 74 (2006) 155435.

17) V. Weidenhof, I. Friedrich, S. Ziegler, and M. Wuttig: J. Appl. Phys. 86 (1999) 5879.

18) S. Y. Kim, S. J. Kim, H. Seo, and M. R. Kim: Jpn. J. Appl. Phys. 38 (1999) 1713.

19) K. P. Chiu, K. F. Lai, and D. P. Tsai: Opt. Express 16 (2008) 13885. 